\title{
COM \\ Engaging with policy practitioners to promote institutionalisation of public participation in science, technology and innovation policy
}

\section{Mitsuru Kudo, Go Yoshizawa and Kei Kano}

\begin{abstract}
This paper is a reflective account of a public participation project the authors conducted in Japan in 2012-2015, as part of the central government's initiative for evidence-based policy-making. The reflection focusses on three key aspects of the project: setting a precedent of involving public participation in policy-making; embedding an official mechanism for public participation in policy-making process; and raising policy practitioners' awareness of public participation. We also discuss why we think engaging with policy practitioners, while problematic in various ways, is and will continue to be important in promoting institutionalised practice of public participation.
\end{abstract}

Keywords

DOI

Background
Participation and science governance; Public engagement with science and technology; Science and policy-making

https://doi.org/10.22323/2.17040801
Papers published in journals on relationships between science and society over the last decade or so suggest that reflecting voices of members of the public in the formation of public policy about science, technology and innovation (STI) has been given increasing recognition in STI policy [e.g. Braun and Könninger, 2018; Emery, Mulder and Frewer, 2015]. In Japan, the government has placed a growing emphasis on the importance of listening to members of the public when making decisions on public policy related to STI. The highest-level policy framework of STI in Japan, namely the Science and Technology Basic Plan, today carries a slogan "Deepening the relationship between STI and society" [Government of Japan, 2016]. It regards coordination among STI policy actors, STI research communities and a broad range of stakeholders as of prime importance in pursuing advancement of STI and economic prosperities in the era of so-called knowledge economy or knowledge-based society. In this context, developing and implementing multi-stakeholder communication mechanisms including public dialogues is mentioned as an important mission for science communication.

It was against this policy background that the authors ran a project for public participation, namely the Framework for Broad Public Engagement in Science, 
Technology and Innovation Policy (PESTI), from October 2012 to September 2015, which is the focus of the present paper. The project was funded and conducted as part of an umbrella initiative ${ }^{1}$ for evidence-based policy-making administered by the Ministry of Education, Culture Sports, Science and Technology (MEXT), which placed a strong emphasis on collaboration between researchers and policy practitioners $^{2}$ so that research findings can have direct relevance and applicability to the actual policy-making processes. Accordingly, like all the other projects conducted under the same initiative, the primary objective of the PESTI project was to develop models and tools for public participation in STI policy-making while simultaneously feeding the research findings to, and responding to the issues identified in, the ongoing process of policy-making on existing policy topics.

Although we were aware that pursuing a direct impact of public participation on ongoing policy-making processes could possibly hinder the legitimacy of its practice [e.g. Irwin, 2006; Wynne, 2006], it seemed to reasonably match with the interest of the PESTI project in promoting institutionalisation of public participation in STI policy-making. Up to the point of the project launch, many among the eighteen project members of the PESTI including the authors had been involved in public dialogues on STI topics. Those dialogues were made available widely across Japan partly in response to the policy call for mechanisms of public participation (mentioned above), and some of the dialogues were designed and implemented with a view to inform relevant policy-making processes with opinions expressed by publics participating in the dialogues. However, such an expected uptake of public voices by the government's policy-making process was rarely observed, and it rather seemed to us that public voices continued to be regarded by policy practitioners as rather marginal or irrelevant to the policy-making process. As practitioners of public dialogues, we saw many participating members of the public disappointed with the lack of established institutional systems to feed their voices into the process of policy-making on STI topics related to the dialogue events in which they had participated. Therefore, we felt the need to promote institutionalisation of public participation so as to bridge the major gap between the policy ideal of open and participatory policy-making and its actual implementation in the existing policy-making process, which drove us to launch the PESTI project with an objective to have an impact on the actual policy-making process.

Besides our recognition of the gap between the lack of meaningful institutional linkage between public dialogues and policy-making processes, we had another concern about the government's decreasing appetite for institutionalised participatory mechanisms. Soon after the Democratic Party of Japan came into power in 2009, it launched several projects towards open and participatory governance of various public policy themes including the government's project

\footnotetext{
${ }^{1}$ The initiative is titled Science for RE-designing Science, Technology and Innovation Policy (SciREX). SciREX was launched in late 2011 and is still continuing. For details, see https:/ / scirex.grips.ac.jp/en/ (visited on 8 September 2018).

${ }^{2}$ In this paper, the term "policy practitioners" refers to those working professionally on public policy in various capacities. They include government officials working in ministries of the central government, policy officers working for scientific research institutions, and administrators of funding agencies.
} 
spending review, ${ }^{3}$ education, ${ }^{4}$ and energy. ${ }^{5}$ Taken together, it was probably not too optimistic back then for us to expect the culture of policy-making would shift towards participatory governance, very slowly but gradually. However, once the former ruling party, namely the Liberal Democratic Party, came back in power in late 2012, many of the previous initiatives taken by the Democratic Party of Japan were brought to an end, and the political mood for public dialogues and participation generally faded away. Having seen such a loss of momentum towards public participation, we had a growing concern that the political mood towards open, participatory policy-making would be lost. It drove us to launch a project of public participation with a focus on promoting its institutionalisation in the STI policy-making process.

Promoting institutionalisation of public participation through engaging with policy practitioners
The PESTI project set its primary objective as presenting the policy-making arena with a convincing case that public participation should be institutionalised. Towards this objective, our strategy was to focus on engaging with policy practitioners. In our view, policy practitioners are the ones playing a crucial role as mediators between public participation events and the policy-making process. In order for voices of members of the public from dialogue events to be connected to the policymaking process, those voices have to be recognised and handled properly by policy practitioners. They are in the position to review outcomes from public participation events, interpret public voices, and feeding them into the administrative systems of policy-making. Instead, they can possibly ignore them. In this sense, our recognition of their role in institutionalising public participation was much the same as that in preceding studies focussed on engaging policy practitioners with public participation [e.g. Escobar, 2014; Pallett, 2015; Russell, 2013]. It seemed essential to us to actively engage with them to find ways to collaboratively work towards more robust policy-making systems with public participation.

Upon launching the PESTI project, we set three inter-related working goals to achieve through engaging policy practitioners with ideas and actual practice of public participation. Below we give a descriptive account to these three goals respectively, detailing why the goals were important, what kinds of activities we conducted, and how the three goals were achieved in the end.

\section{Setting a precedent of involving public participation in STI policy-making}

One of the three working goals was to set a precedent of STI policy-making with public voices officially taken into account through participatory mechanisms. The PESTI project placed an emphasis on demonstrating the positive values and

\footnotetext{
${ }^{3}$ With the project title Jigyou Shiwake, it aimed to increase accountability and transparency of budgetary decision-making by inviting external experts to the review panels and publicising the review process.

${ }^{4}$ The MEXT built an experimental platform of deliberative decision-making on education policy by combining face-to-face meetings and online virtual meetings [The Ministry of Education, Culture, Sports, Science and Technology (MEXT), 2010].

${ }^{5}$ The project was a type of public participation in energy policy, delivered in the format of Deliberative Polling [Fishkin and Farrar, 2005] on a national scale, in response to the nuclear powerplant accidents in Fukushima in 2011 and the resultant debate over sustainability of nuclear power [Mikami, 2015]. For various possible reasons including the change of government, the results from the Deliberative Polling were not taken into account in related policy-making processes in the end.
} 
relevance of public participation to STI policy-making from the policy practitioners' perspectives, in addition to pursuing a good practice of public dialogues. Our expectations were that if we could set at least one precedent of demonstrably successful STI policy-making with public participation as its key component, it would be easier for policy practitioners to build other cases of policy-making with public participation by following the administrative procedures set in the precedent.

To achieve this working goal, it was crucial for us to identify an STI policy topic that would give public participation the best chance to become integrated into the policy-making process. We sought an STI policy topic that was positioned at an early stage of its formation so as to avoid having public voices misused by the policy-making process to give ostensible legitimacy to unchangeable decisions. From the beginning of the PESTI project, we talked to interested policy practitioners and had a series of discussions to review emerging policy agendas for the coming months, with regard to their potential to allow public participation to have a constructive impact on the policy-making process in its "upstream" phase [Wilsdon and Willis, 2004]. In the end, we agreed to set JAPAN Vision 2020 as the principal policy topic for our PESTI project to work on. JAPAN Vision 2020 was a policy topic of the MEXT to create a vision of the Japanese society towards the Tokyo Olympic and Paralympic Games in 2020 and beyond, with a focus on how STI would help the society achieve the vision [The Ministry of Education, Culture, Sports, Science and Technology (MEXT), 2014]. It was claimed to be across bureaus, departments and divisions of the MEXT, and the Vision was going to be positioned as a guiding policy for a wide range of STI policies formed within the ministry. Therefore, JAPAN Vision 2020 seemed apt as the STI policy topic for the PESTI project to focus on.

Once our target policy was set to be JAPAN Vision 2020, we began collecting information to ensure that opinions and voices expressed in our public dialogue events would be more likely fed into the actual policy-making process of JAPAN Vision 2020. As one of the main foci of the PESTI project, we reached out to wider publics through our diverse public participation events by employing existing and newly developed models of participants segmentation [Goto et al., 2014; Goto, Kudo and Kano, 2015; Kano, 2014; Suga et al., 2017; Yoshizawa et al., 2016]. Outcomes from these events were delivered to the policy-making process in accordance with the information collected provided by the policy practitioners, which included which policy practitioners were going to play key roles in shaping the policy, about what aspects of the policy they were interested in hearing public voices, in what forms the public voices should be packaged, and by when they had to be presented to the policy-making process. Some policy practitioners were very helpful to us not only in finding the information, but also in efficiently planning, designing and implementing the series of public dialogues and other necessary activities. ${ }^{6}$ Furthermore, they acted as a liaison between the policy-making arena and our public dialogues, enabling us to deliver voices from the participants in our public dialogues directly to the right policy practitioners, rather than communicate public voices to the policy-making arena without knowing who would receive and handle the public voices to what ends. They also provided us with feedback regarding how the participants' voices from the PESTI project's public dialogues had been received by the policy-making process.

\footnotetext{
${ }^{6}$ One of the policy practitioners was appointed as a member of the ministry's working board specially convened for JAPAN Vision 2020.
} 
In January 2014, the MEXT officially announced the completed JAPAN Vision $2020 .^{7}$ In its official document we could recognise some of the public voices from the dialogue events of PESTI appearing as part of the policy. In addition, the policy document also refers to the PESTI project as one of the contributors to the making of JAPAN Vision 2020 from the public participation perspective. This shows the outcome of the PESTI project's work on JAPAN Vision 2020 was given positive recognition by the MEXT.

\section{Embedding an official mechanism for public participation in policy-making process}

The second working goal was associated directly with the institutionalisation of public participation. It was to embed an official mechanism for practising public participation in the government's administration system of STI policy-making. What we mean by "mechanism" in this context would possibly take various different forms. For example, it could be a written statement issued officially by the government that acknowledges the need for public participation in STI policy-making. Alternatively, it could be newly set up funding schemes or professional positions designated specially for administrating public participation in STI policy-making. The point of embedding an official mechanism for public participation in the policy-making process, however minor the mechanism would be, was that once it was embedded officially in the formal process of the government administration, it would unlikely be disregarded or eliminated from the system. In other words, we were afraid that once the PESTI project finished after its funding period of three years, and/or once those policy practitioners collaborating with our public participation project were rotated to another position, the progress we had made on the policy-making process towards public participation would be critically undermined. Therefore, in our view, leaving something institutionally recognisable, acknowledgeable and sustainable would be essential.

Towards this second working goal, we focussed on the concept of "interactive policy-making" and promoted it as the new mode of STI policy-making the MEXT should incorporate to better respond to the changing nature of STI. While we were working on JAPAN Vision 2020, we prepared a briefing report on interactive policy-making for interested policy practitioners, in which we cited academic literature [e.g. Torfing and Triantafillou, 2011] and some recent preceding examples of policy formation through the interactive policy-making approach. We also regularly talked to the interested policy practitioners about why such change was anticipated in the system of STI governance today.

As a result of our project's promotion of the concept of interactive policy-making, the MEXT set up the Office for Interactive Policy Making in the Policy Division of Minister's Secretariat in October 2014. Although the Office is physically nothing more than a small office space located in the MEXT building, it is administratively "a permanent space" [The Ministry of Education, Culture, Sports, Science and Technology (MEXT), 2015, p. 329] and is given formal recognition as an important element of policy-making with a mission to facilitate multi-stakeholder collaboration and public dialogues. Especially given our concern about the then

\footnotetext{
${ }^{7}$ The document of JAPAN Vision 2020 is available on the MEXT website, both in the original language (Japanese) (http:/ / www.mext.go.jp/b_menu/houdou/26/01/1343297.htm) and in English (http://www.mext.go.jp/en/news/topics/detail/1372653.htm) (both visited on 8 September 2018).
} 
government's declining interest in public participation (mentioned earlier), this outcome was remarkable.

\section{Raising policy practitioners' awareness of public participation}

The third working goal was to raise policy practitioners' awareness of public participation. It may sound broad and less concrete in comparison to the first two working goals, but nevertheless was an essential working goal for the PESTI project. It was our project's expectation that drawing attention of policy practitioners to public participation and raising their awareness of its potential and actual values to STI policy-making would give fresh impetus to the delayed institutionalisation of public participation.

From the beginning of the PESTI project, we made a variety of opportunities to discuss public participation in STI policy-making with policy practitioners. They were mainly from the MEXT and the Ministry of Economics, Trade and Industry (METI), two of the ministries most closely associated with STI policy in Japan. One noticeable example of our engagement activities was what we labelled as Policy Design Workshop [Yoshizawa, 2013]. It was organised as a series of informal workshops, attended by policy practitioners from various ministries and STI research institutions, as well as policy researchers. In order to maintain a casual atmosphere, we used cafes and rented casual workspaces as a venue and served light refreshments. Policy Design Workshops worked as a platform for both researchers and practitioners of STI policy to learn together the values and meanings of public participation in policy-making, and the ways in which it could be institutionally incorporated into the existing policy-making process. Policy Design Workshops were delivered at an early stage of the PESTI project, which paved the way for further collaborative works between interested policy practitioners and the PESTI project on JAPAN Vision 2020 and other specific STI policy topics.

Another noticeable example of our engagement with policy practitioners was a public forum organised halfway through the project. The forum was designed to reflect on the process and outcomes of public participation through the PESTI project on JAPAN Vision 2020. For this public forum, we invited the key policy practitioner collaborating with PESTI and the participants in our preceding public dialogues on JAPAN Vision 2020. In the forum, the participants shared with the policy practitioner their frank opinions about their experience in becoming part of the public participation activities on JAPAN Vision 2020, and exchanged their views on to what degree and in what forms public participation should be institutionalised in the process of STI policy-making.

Besides the Policy Design Workshops and the public forum, the PESTI project managed to organise a number of discussion opportunities with policy practitioners throughout the course of its funding period of three years. We regularly talked to interested policy practitioners to discuss and share our views on STI policy-making and public participation. Occasionally those conversations took place only in the margin of official meetings with them, but those conversations were highly valuable in developing mutual understanding in a frank manner. In addition, the discussion opportunities also helped the PESTI project gather direct feedback from policy practitioners to the project. Evaluating the impact of those 
engagement activities on the awareness of the policy practitioners about public participation was not an easy task. Yet, during the PESTI project, we occasionally heard from the policy practitioners, who had participated in Policy Design Workshops and our other dialogue events, about positive impressions they had about public participation as a necessary mechanism for STI policy-making. This indicated that our engagement activities caught their attention and nudged them to think constructively about how they could possibly connect public participation with their policy-making practice.

Limitations of our approach

As presented so far, we managed to feed public voices from the public dialogue events into the actual process of policy-making of an STI policy topic, namely JAPAN Vision 2020. We also informed the policy practitioners who had participated in activities organised by the PSETI project about the interactive policy-making approach, which led to the launch of the Office for Interactive Policy-making within the MEXT. Furthermore, we raised the policy practitioners' awareness about public participation through Policy Design Workshops and public dialogue events, where we discussed with them the meanings and necessity of public participation. In addition to these outcomes, the policy practitioners expressed satisfaction with what they had experienced in and learnt from their own commitment to the PESTI project and its associated public participation activities. These all indicate that the PESTI project had made some progress towards institutionally embedding public participation in the official process of policy-making about STI.

Having said this, we should bear in mind that both policy practitioners' views on public participation and their organisational systems of policy-making would not change over a short period of time. Put differently, we should avoid too simplistic a judgement on an immediate impact of our intervention on the policy-making towards public participation.

In addition, we also should note that our project's emphasis on bringing an actual difference to the existing system of STI policy-making necessarily leads us to question the autonomy of our public engagement activities from structural constraints of the policy-making process. As detailed in an earlier section, we were most concerned to present public voices from our public dialogue events so that they could be seen by policy practitioners as relevant to and necessary for their policy-making practice. Consequently, our framing of public dialogue events and our reporting of participants' voices to the policy-making process were both under significant influence of how the policy practitioners we were collaborating with would make sense of public voices in their own policy-making contexts [cf. Akiya et al., 2014]. In other words, we framed our public dialogues primarily for our project's strategic goal of setting a precedent of involving public participation in policy-making, rather than in the way that would have illuminated what vision the participants actually had in mind about the future vision of the society.

Engaging policy practitioners as a way forward
While fully acknowledging those issues and difficulties in connecting public dialogues with the actual policy-making process through collaboration with policy practitioners, we would still highly value engaging interested policy practitioners 
with public participation as an important means to promote institutionalisation of public participation. This is because, as discussed below, there seem to be many policy practitioners who are the keenest to improve the process of policy-making.

What we found about policy practitioners through the PESTI project was that many of them were unsatisfied, and occasionally frustrated, with the existing practice of policy-making. In their views, established figures with authoritative power in STI fields would often dominate the process of shaping STI policy. Similarly, senior policy practitioners' intuition and anecdotal evidence would be given more recognition than relevant expertise and scientific evidence provided by those less established. This was partly why some of the frustrated policy practitioners saw in public participation the potential to bring an actual change to the policy-making. For example, one of the policy practitioners collaborating with the PESTI project told us that the key aspect of public participation for them would be its emphasis on opening up the closed process of the current policy-making practice to external actors including members of the general public. Along similar lines, other interested policy practitioners saw public participation as providing policy practitioners, particularly junior officials, with opportunities to work collaboratively with officials from other ministries, external stakeholders, experts and professionals in related fields. In this sense, their uptake of public participation was not necessarily driven by their resonance with the conceptual ideals of public participation. Yet, in the sense that they were aiming at challenging the policy-making system for broader participation, more collaboration and openness through institutionalising public participation in the policy-making process, we worked towards the same goal. Therefore, when they were invited to work together with our PESTI project on promoting public participation in the policy-making process, they were not passive recipients of information about public participation. Rather, together with our project members, they were actively searching for what public participation could possibly bring to their everyday practice of policy-making so that their capacity for designing and implementing alternative policy-making processes could be expanded.

\section{Conclusion}

Putting together what we have discussed so far, we argue for the necessity for researchers and practitioners of science communication to continue, or start, creating a space to share with policy practitioners across different bureaus, departments, divisions and ministries, where we can collaboratively explore what meanings public participation can possibly have in different policy-related contexts. What we have learnt from collaboratively working with policy practitioners is that although policy practitioners with interest in public participation are embedded in a strictly institutionalised process of policy-making and are under strong time pressure and administrative constraints, they fully exercise their agency to make their own decisions throughout the process of policy-making to challenge the system. We have also come to think that the more we communicate and learn from each other, the more we can collaborate in taking on the shared challenge of opening up the existing policy-making process to wider participation of different actors who are currently not institutionally recognised as an important part of the process.

It will most likely take years before we can observe any change in the actual practice of policy-making achieved by such communication and collaboration. 
Researchers and funding bodies providing necessary resources to such projects will need to be prepared to face this difficulty in capturing the outcome of engaging with policy practitioners, and should avoid short-sighted judgement and evaluation of it.

Acknowledgments

\section{References}

The PESTI project (October 2012 to September 2015) was funded by the Research Institute of Science and Technology for Society of the Japan Science and Technology Agency, under one of its funding programmes titled "Science of Science, Technology and Innovation Policy".

Akiya, N., Takanashi, K., Mizumachi, E., Kudo, M. and Kano, K. (2014). 'As any person, what to speak: a way of handling speaker's identity in workshop'. Japanese Journal of Science Communication 15 (06), pp. 107-122. URL: http://hdl . handle.net/2115/56444.

Braun, K. and Könninger, S. (2018). 'From experiments to ecosystems? Reviewing public participation, scientific governance and the systemic turn'. Public Understanding of Science 27 (6), pp. 674-689. https://doi.org/10.1177/0963662517717375.

Emery, S. B., Mulder, H. A. J. and Frewer, L. J. (2015). 'Maximizing the policy impacts of public engagement: a European study'. Science, Technology, E Human Values 40 (3), pp. 421-444. https: //doi .org/10.1177/0162243914550319.

Escobar, O. (2014). 'Upstream public engagement, downstream policy-making? The brain imaging dialogue as a community of inquiry'. Science and Public Policy 41 (4), pp. 480-492. https://doi.org/10.1093/scipol/sct073.

Fishkin, J. and Farrar, C. (2005). 'Deliberative polling: from experiment to community resource'. In: The deliberative democracy handbook: strategies for effective civic engagement in the twenty-first century. Ed. by J. Gastil and P. Levine. San Francisco, CA, U.S.A.: Jossey-Bass, pp. 68-79.

Goto, T., Kudo, M. and Kano, K. (2015). 'Measuring representativeness of participants in public engagement events'. Japanese Journal of Science Communication 17 (07), pp. 3-19. URL: http://hdl . handle.net/2115/59575.

Goto, T., Mizumachi, E., Kudo, M. and Kano, K. (2014). 'Verification on the utility of using the segmentation method developed in Australia to assess audiences of science and technology events'. Japanese Journal of Science Communication 15 (06), pp. 17-35. URL: http://hdl . handle.net/2115/56441.

Government of Japan (2016). Science and technology basic plan. Japan. URL: http://www8.cao.go.jp/cstp/english/basic/5thbasicplan.pdf.

Irwin, A. (2006). 'The Politics of Talk'. Social Studies of Science 36 (2), pp. 299-320. https://doi.org/10.1177/0306312706053350.

Kano, K. (2014). 'Toward achieving broad public engagement with science, technology and innovation policies: trials in JAPAN Vision 2020'. International Journal of Deliberative Mechanisms in Science 3 (1), pp. 1-23. https://doi.org/10.4471/demesci.2014.12.

Mikami, N. (2015). 'Public participation in decision-making on energy policy: the case of the "National Discussion" after the Fukushima accident'. In: Lessons from Fukushima: Japanese case studies on science, technology and society. Ed. by Y. Fujigaki. Cham, Switzerland: Springer International Publishing, pp. 87-122. https://doi.org/10.1007/978-3-319-15353-7_5. 
Pallett, H. (2015). ‘Public participation organizations and open policy: a constitutional moment for British democracy?' Science Communication 37 (6), pp. 769-794. https://doi.org/10.1177/1075547015612787.

Russell, A. W. (2013). 'Improving legitimacy in nanotechnology policy development through stakeholder and community engagement: forging new pathways'. Review of Policy Research 30 (5), pp. 566-587. https://doi.org/10.1111/ropr.12037.

Suga, M., Suzuki, N., Fujiwara, N., Yoshizawa, G., Kudo, M. and Kano, K. (2017). 'Development of a new segmentation for broad public engagement in science, technology and innovation policy: from an opinion poll on science, technology and innovation'. Japanese Journal of Science Communication 22 (12), pp. 3-13. URL: http://hdl. handle.net/2115/67955.

The Ministry of Education, Culture, Sports, Science and Technology (MEXT) (2010). 2010 White Paper on Education, Culture, Sports, Science and Technology. URL: http://www.mext.go.jp/b_menu/hakusho/html/hpab201001/1326588.ht $\mathrm{m}$ (visited on 8th September 2018).

- (2014). White Paper on Science and Technology 2014. URL: http: //www . mext.go .jp/en/publication/whitepaper/title03/detail03/1372828.htm (visited on 8th September 2018).

- (2015). White Paper on Science and Technology 2015. URL: http: //www . mext.go .jp/en/publication/whitepaper/title03/detail03/1372827.htm (visited on 8th September 2018).

Torfing, J. and Triantafillou, P. (2011). Interactive Policy Making, Metagovernance and Democracy. Colchester, U.K.: ECPR Press.

Wilsdon, J. and Willis, R. (2004). See-through Science: Why Public Engagement Needs to Move Upstream. London, U.K.: Demos.

Wynne, B. (2006). 'Public Engagement as a Means of Restoring Public Trust in Science - Hitting the Notes, but Missing the Music?' Community Genetics 9 (3), pp. 211-220. https://doi.org/10.1159/000092659.

Yoshizawa, G. (2013). 'Policy design workshop: a space for knowledge exchange between practitioners and researchers'. In: Proceeding of Japan Society for Research Policy and Innovation Management 2013. (Tokyo, Japan, 2nd-3rd November 2013), pp. 917-920. URL: http://hdl . handle.net/10119/11857.

Yoshizawa, G., Suga, M., Kudo, M., Mizumachi, E. and Kano, K. (2016). 'Understanding the plurality of public interests for open strategy in science and innovation'. In: Proceeding of OECD Blue Sky Forum on Science and Innovation Indicators 2016. (Ghent, Belgium, 19th-21st September 2016).

URL: https://www. oecd.org/sti/032\%20-\%20P-Seg_final.pdf.

\section{Authors}

Mitsuru Kudo, $\mathrm{PhD}$, is an associate professor at the Center for the Study of Co* Design, Osaka University. Over the last ten years he has been working on several research projects on various topics in and around science communication, including science literacy, public participation, community engagement and science communication policy. He also teaches science communication in the Programme for Education and Research on Science and Technology in Public Sphere, a postgraduate minor programme delivered at the Osaka University. E-mail:mkudo@cscd.osaka-u.ac.jp. 
Go Yoshizawa, PhD is Research Fellow at Work Research Institute (AFI), OsloMet — Oslo Metropolitan University. Designing, organising and facilitating dozens of transdisciplinary projects, meetings and workshops, he has committed himself to future-oriented strategic intelligence in science and technology policy including technology assessment, foresight and responsible research and innovation. His current research interests include knowledge policy and management, intermediary organisations, and non-verbal communication for wider engagement in science and innovation. E-mail: gyosh@oslomet.no.

Kei Kano, $\mathrm{PhD}$, is an associate professor at the Science Communication Laboratory, Graduate School of Education, Shiga University, Japan. He has worked widely in science communication or public engagement for grade school students, adults, and early-career scientists. Dr. Kano is interested in inclusiveness of science communication or public engagement activities. E-mail: kkano@edu.shiga-u.ac.jp.

How to cite

Kudo, M., Yoshizawa, G. and Kano, K. (2018). 'Engaging with policy practitioners to promote institutionalisation of public participation in science, technology and innovation policy'. JCOM 17 (04), N01. https:/ / doi.org/10.22323/2.17040801. 